\title{
Key attributes of integrated community-based youth service hubs for mental health: a scoping review
}

\author{
Cara A. Settipani ${ }^{1}$, Lisa D. Hawke1,3, Kristin Cleverley ${ }^{1,2}$, Gloria Chaim ${ }^{1,3}$, Amy Cheung ${ }^{3,4}$, Kamna Mehra ${ }^{1}$,
} Maureen Rice ${ }^{5}$, Peter Szatmari ${ }^{1,3,6}$ and Joanna Henderson ${ }^{1,3^{*}}$ (D)

\begin{abstract}
Background: Community-based, integrated youth service hubs have the potential to address some of the longstanding issues with mental health services for youth, including problems with access and system fragmentation. Better understanding of these approaches, particularly efforts to create a single point of entry to comprehensive, evidence-based services through youth service hubs, is needed to help guide future implementation and evaluation. This scoping review identifies the key principles and characteristics of these models of care, as well as the state of the literature, particularly with regard to implementation and replicability.
\end{abstract}

Method: Electronic databases and grey literature sources were searched for material from 2001 to 2019, with diverse search terms capturing the concept of "integrated" or "one-stop shop" youth mental health services. Title/abstract and full text review were conducted, as well as additional focused searching. After screening 4891 texts at the title/ abstract level and 496 at the full-text level, 110 documents were included for data extraction.

Results: Several integrated care hub models for youth mental health services and related frameworks were identified internationally, largely in high-income countries. Common principles included an emphasis on rapid access to care and early intervention, youth and family engagement, youth-friendly settings and services, evidence-informed approaches, and partnerships and collaboration. Program characteristics also revealed similarities (e.g., providing evidence-informed or evidence-based services in youth-friendly spaces), with some differences (e.g., care coordination methods, types of service providers), potentially attributable to lack of available information about key ingredients. Outcome research was limited, with few rigorous evaluations of youth outcomes. Moreover, sufficient information for replication, community evaluation of feasibility or actual implementation was rarely provided.

Conclusion: Internationally, integrated youth service hubs were found to share common key principles, while providing comprehensive services to youth with mental health difficulties. There is a great need for common language and measurement framework to facilitate replication, rigorous evaluation of outcomes, knowledge exchange, and dissemination of findings.

Keywords: Integrated care hubs, Mental health, Addiction, Youth, Young adults

\footnotetext{
*Correspondence: Joanna.henderson@camh.ca

${ }^{1}$ Margaret and Wallace McCain Centre for Child, Youth and Family Mental

Health, Centre for Addiction and Mental Health, Toronto, ON, Canada

Full list of author information is available at the end of the article
} 


\section{Introduction}

Mental health problems commonly start during childhood or adolescence, with about half of all lifetime cases starting by ages $14-15$ and three-quarters by ages 18-24 [1, 2]. Worldwide, approximately 10-20\% of children and adolescents experience mental health disorders $[3,4]$ and cumulative lifetime prevalence approaches $50 \%$ among adolescents and young adults in developed countries $[1,5,6]$. Mental health problems are also associated with high societal burden [7, 8]. Despite the clear need for mental health services, youth often do not receive evidence-based services in a timely, effective manner $[9,10]$. Longstanding problems with youth mental health service provision, including system fragmentation, poor access to evidence-based services, discontinuation of support at the transition to adulthood and lack of an informed developmental perspective, could be effectively addressed by integrated care approaches offered across the adolescent and young adult developmental stages [11, 12]. Indeed, models of care that bring traditionally separate services together into one community-based setting to meet youth's unique needs and span the transition to adulthood have recently gained the attention of researchers, service providers, and policymakers [13-15].

Integrated community-based youth service hubs (ICYSHs) reflect models of care that provide comprehensive, youth-focused services, including mental health services, health and other community and social services in a single community-based setting, sometimes referred to as "one-stop shops", with "youth" defined to include both adolescents and young adults. Literature reviews of medical and behavioral health integration in primary care settings have been conducted $[16,17]$ and a meta-analysis suggests benefits [18]. However, although ICYSH models are being adopted internationally $[13,14]$, reviews of these integrated community-based models of care are lacking. One recent exception is a 2017 review by Hetrick et al. [19], which describes select programs and their outcome evaluation efforts. Authors highlight the need to further evaluate outcomes and develop best practice models [19]. They do not, however, examine the common principles across various exemplars of ICYSH. In addition, information on implementation of essential features of ICYSH models is needed to ensure that the components most crucial to their success are included in efforts to replicate the model and evaluate the feasibility and outcomes of the model in different contexts. This review identifies ICYSH models for mental health services, their characteristics, common principles, and aspects critical to implementation and replicability.

\section{Methods}

Search strategy and identification of relevant studies

Scoping review methodology was deemed particularly well-suited to examine the burgeoning area of ICYSHs. Scoping reviews comprehensively identify important concepts in a structured manner [20], including the extent, range, and nature of research activity in areas in which the available range of materials is unknown [21], e.g., when substantial grey literature is expected. The scoping methodology described in the literature was followed [21-23]. For a detailed description of the review methodology, see [24]. An electronic search was conducted in Medline, EMBASE, PsycINFO, CINAHL, ASSIA, and Campbell Collaboration Library and Cochrane Library using a combination of subject heading and text word terms for integration AND mental/behavioral health AND children/adolescents. Work published in English between 2001 and 2019 was included to capture recent developments in ICYSH models. Exclusion criteria included comments or notes, editorials, and letters. A total of 4817 unique citations were retrieved from the initial database search, after removing 1166 duplicates (see Fig. 1 for PRISMA diagram).

A grey literature search was undertaken following a comprehensive checklist [25]. A broad Google Advanced Search was performed and a focused search was conducted on specific youth-relevant mental health sites, resulting in 45 grey literature documents. An additional four documents were gathered by reviewing reference lists. The International Journal of Integrated Care was hand searched, yielding seven more articles. Eighteen additional works (eight articles and 10 grey literature documents) known to the authors were included, resulting in 4891 documents screened.

Inclusion criteria were as follows: presenting problem was mental or behavioral health, substance use concerns, or concurrent disorders; population was children, adolescents, youth, emerging adults, or young adults. The United Nations defines "youth" as the age range spanning from 15 to 24 [26]; the age range accepted for inclusion in the review was flexible to accommodate different definitions retained in the literature. Other inclusion criteria included models of care that brought together multiple service components to improve access to service, service quality, client satisfaction, efficiency [27], or youth outcomes [11], and aimed to make mental health systems or service delivery more fulsome [28]; services were coordinated, co-located (multiple types of services beyond mental health, all in a single setting), and community-based (not hospital, primary care, or school based). Non-research literature (e.g., policy documents) was also included to capture a broad range of work.

Titles and abstracts were independently screened by two raters, with project lead consultation to resolve 


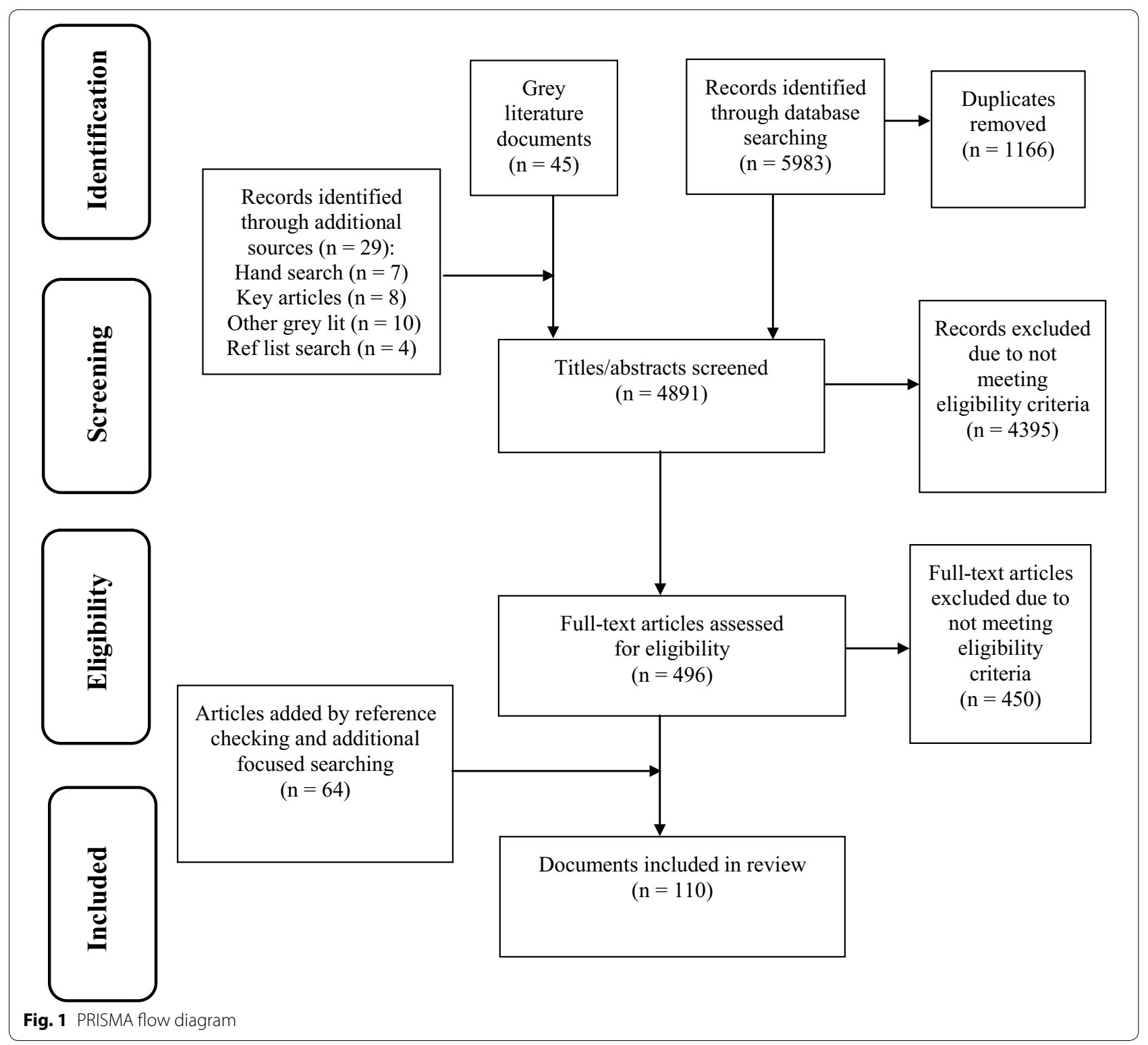

discrepancies. Covidence [29], the Cochrane-recommended systematic review software, was used for title/ abstract and full text review. Reasons for exclusion were: conference presentations presented $>3$ years ago; adults or infants only $($ age $<2)$; not mental or behavioral health; not linked/integrated services; only hospital consultation-liaison services; only combination therapymedication in a randomized control trial. Following title/ abstract screening, 496 full-text articles were examined; of them, 450 documents were ineligible (see Fig. 1). At this stage, the authors checked references of included studies for other relevant work and conducted a focused search for related documents, adding 64 documents; 110 documents were ultimately included for data extraction. Information was extracted from these documents using a data extraction tool which was iteratively modified based on the knowledge gathered during the scoping review process (see published scoping review protocol for more information [24]). Members of the research team repeatedly read the extracted data, returning to the full text for context, in order to synthesize the findings by consensus. Following extraction, data were reviewed to identify unique programs and examine the extent of information available for each program.

\section{Results}

In total, 87 journal articles and 23 grey literature documents were included in the review. The primary purpose of journal articles was: description of ICYSH model, 30; model research, 42; implementation descriptions, 9; RCT 
protocol, 1; position papers, 4; review, 1. The diverse grey literature included: government-commissioned reports, reviews and service frameworks, 5 ; information related to a program model, efforts, and progress, 6; informal program information, 4; in-depth program descriptions/reports, 3; independent program evaluations, 4; model recommendations, 1. In all, 94 documents described components of 8 unique programs (Table 2). Insufficient information to meaningfully review was provided about 11 models, resulting in their exclusion from the detailed analysis in this report, although they are described in summary Table 1.

\section{Summary of models reviewed}

Multiple international ICYSH models were identified (Table 1). Although literature was not restricted by country of origin, the documents included in the review were found to be based on ICYSH from high-income countries. Programs include: headspace and Orygen Youth Health in Australia, Jigsaw in Ireland, Forward Thinking Birmingham (formerly Youthspace) in the United Kingdom, Youth One Stop Shops in New Zealand, and YouthCan IMPACT, Foundry, and ACCESS Open Minds in Canada. See Table 1 for information on all models identified.

The Australian headspace model aims to establish a multidisciplinary, primary mental health services model that is highly accessible and specialized to meet the core needs of young people [30, 31]. It also aims to bridge mental health service gaps through co-location and common clinical governance. A key principle is that youth are not turned away from services based on severity or diagnosis. headspace seeks to provide early intervention within an enhanced primary care one-stop shop that is closely linked to local specialist services, schools, and other communitybased organizations [14]. As of early 2019, there are a total of 110 headspace centers established in Australia [32].

Orygen Youth Health in Australia evolved out of the Early Psychosis Prevention and Intervention Centre (EPPIC) model [14]. This service seeks to bridge the gap between child/adolescent and adult mental health services in the Melbourne area [33]. Four specialized clinics focus on youth with first episode psychosis, mood disorders, emerging borderline personality disorder, and youth at ultra-high risk for a psychotic disorder [14]. Each clinic provides a full complement of interventions over a 2-year period of care. A youth access team provides triage, assessment, and crisis response $24 \mathrm{~h}$ a day, 7 days a week, as well as community and home-based services [14].

Headstrong, the National Centre for Youth Mental Health in Ireland, established Jigsaw [34] to integrate supports and services for young people through community capacity building, with 13 centers currently providing evidence-based services [35]. Key efforts include engaging young people in service design and planning to improve responsivity to youth needs and minimize stigma, building an integrated community network, improving the availability of programs that teach resilience and core competencies (e.g., interpersonal skills), identifying those at risk for mental health problems earlier, ensuring clearly defined pathways to care, and engaging community leaders $[34,36]$.

In England, Forward Thinking Birmingham advocates prevention, choice, and personalized care and offers youth a range of new services and facilities; this model will likely serve as a United Kingdom template for additional youth mental health service reform efforts [13]. Forward Thinking Birmingham is based on the success of pilot initiative Youthspace, which explored effective clinical approaches among youth up to age 25 [37]. Goals included providing rapidly responsive, youthfriendly early intervention services and utilizing a range of treatments.

New Zealand's Youth One Stop Shops were developed independently in response to local needs and opportunities supported by relationships with other providers and funders, though they share a common goal of promoting youth access to healthcare and social services [38]. In 2009, there were at least 14 Youth One Stop Shops across the country, providing a range of youth-friendly, holistic services at little or no cost to the youth.

Several ICYSH efforts are underway in Canada. ACCESS Open Minds is implementing 14 ICYSHs in urban and rural areas across Canada [13, 39, 40]. The multi-stakeholder ACCESS network includes youth, families, community organizations, service providers, researchers, and policy makers, and provides rapid access to evidence-informed, youth-friendly services appropriate for varying levels of need [40, 41]. Foundry is a British Columbia model that co-locates health and social services through a network of centers and e-health services [42]. By supporting communities to develop youth-friendly ICYSH storefronts, working with provincial resources, and linking to specialized services and community agencies, Foundry aims to strengthen a comprehensive system of care for youth and young adults provincially and improve access to mental health, substance use, and primary care services [43]. YouthCan IMPACT in Toronto involves the development, implementation and evaluation of an Integrated Collaborative Care Team (ICCT) model consisting of linked components that are co-located within community-based, youth-friendly ICYSHs [44].

\section{Model characteristics Populations}

See Table 2 for a summary of the characteristics of ICYSH models. Many programs focused on adolescence through 







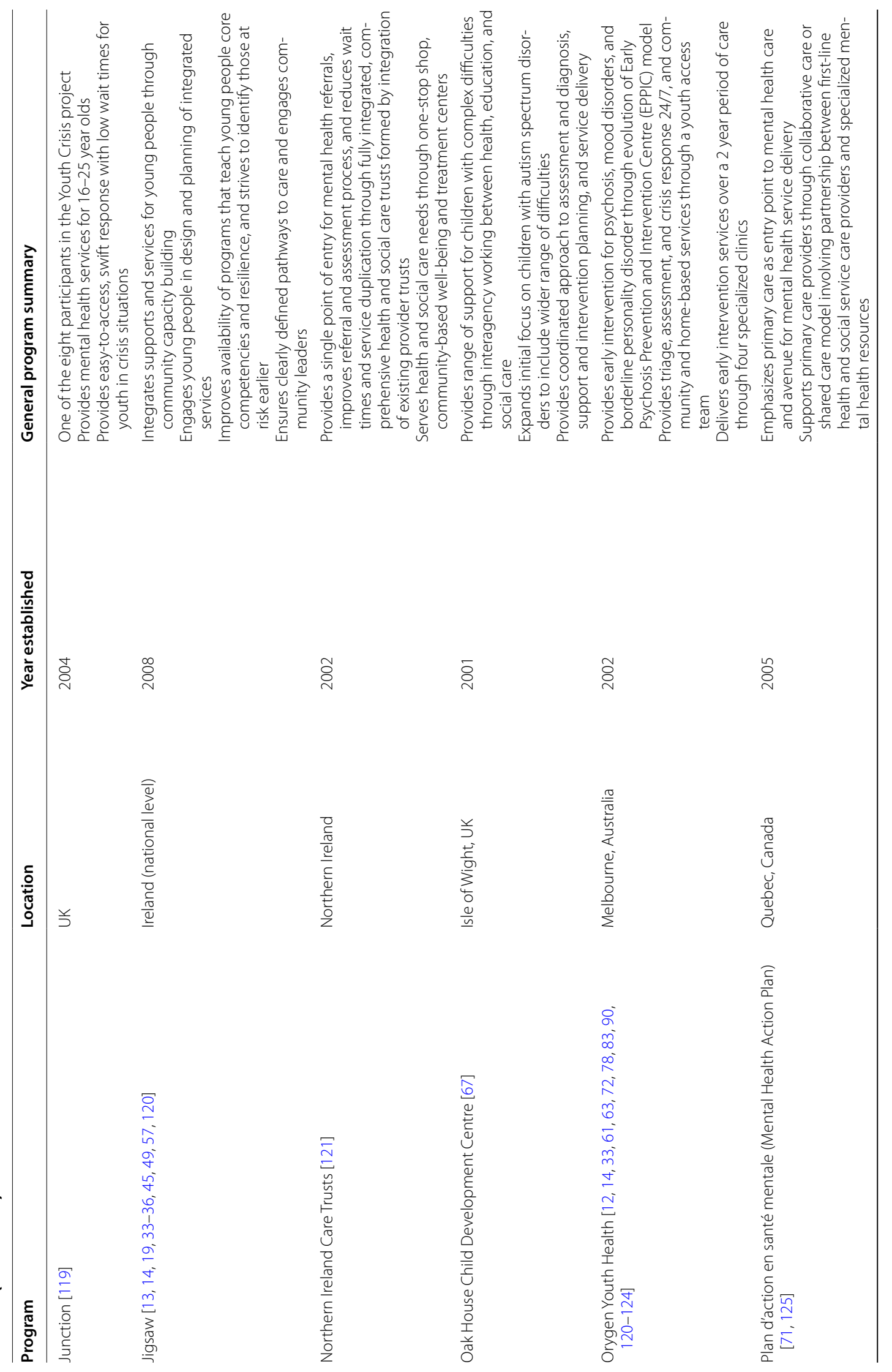


Settipani et al. Int J Mene Health Syst <wide> <wide>(2019) 13:52

Page 7 of 26

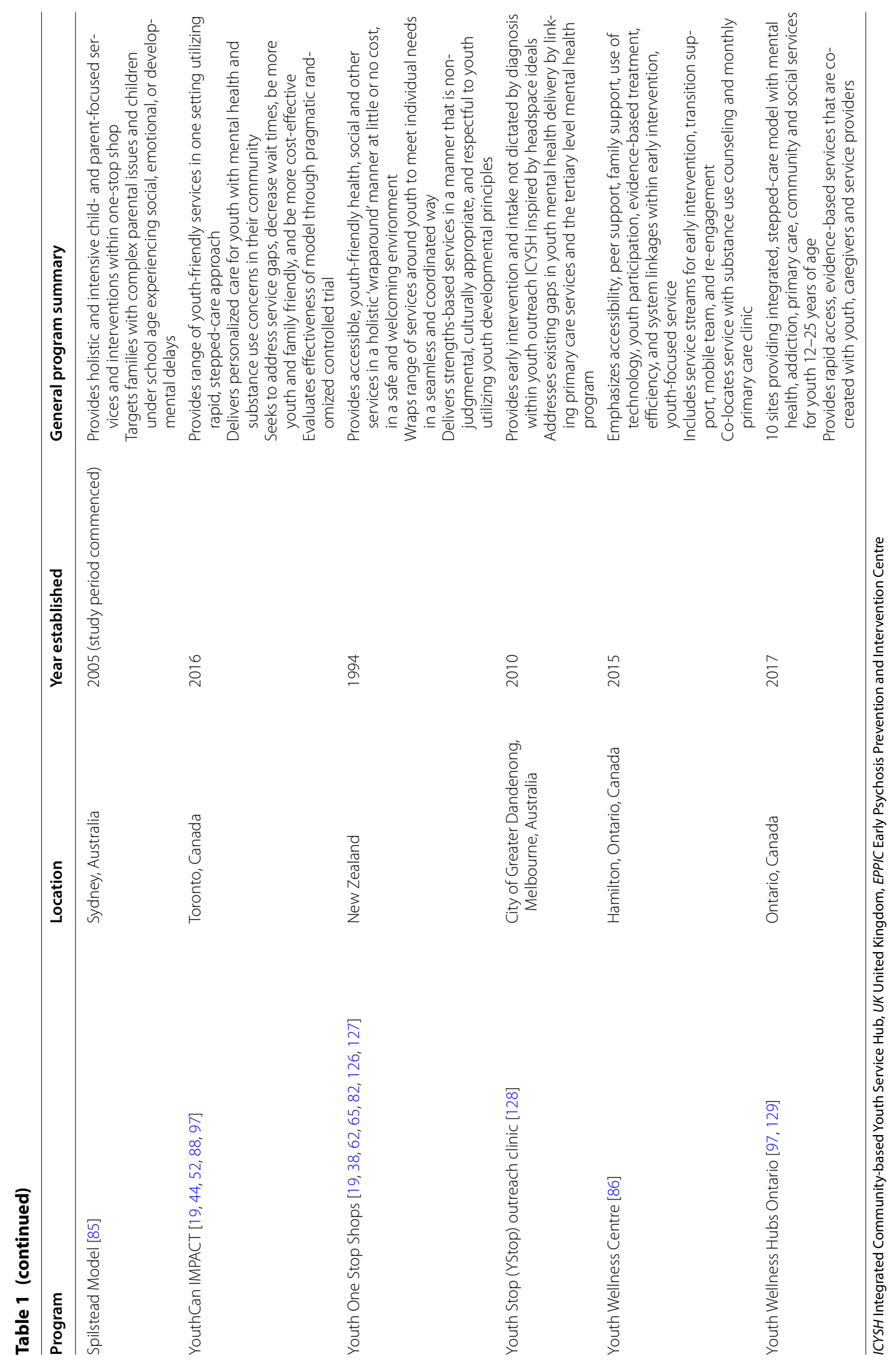




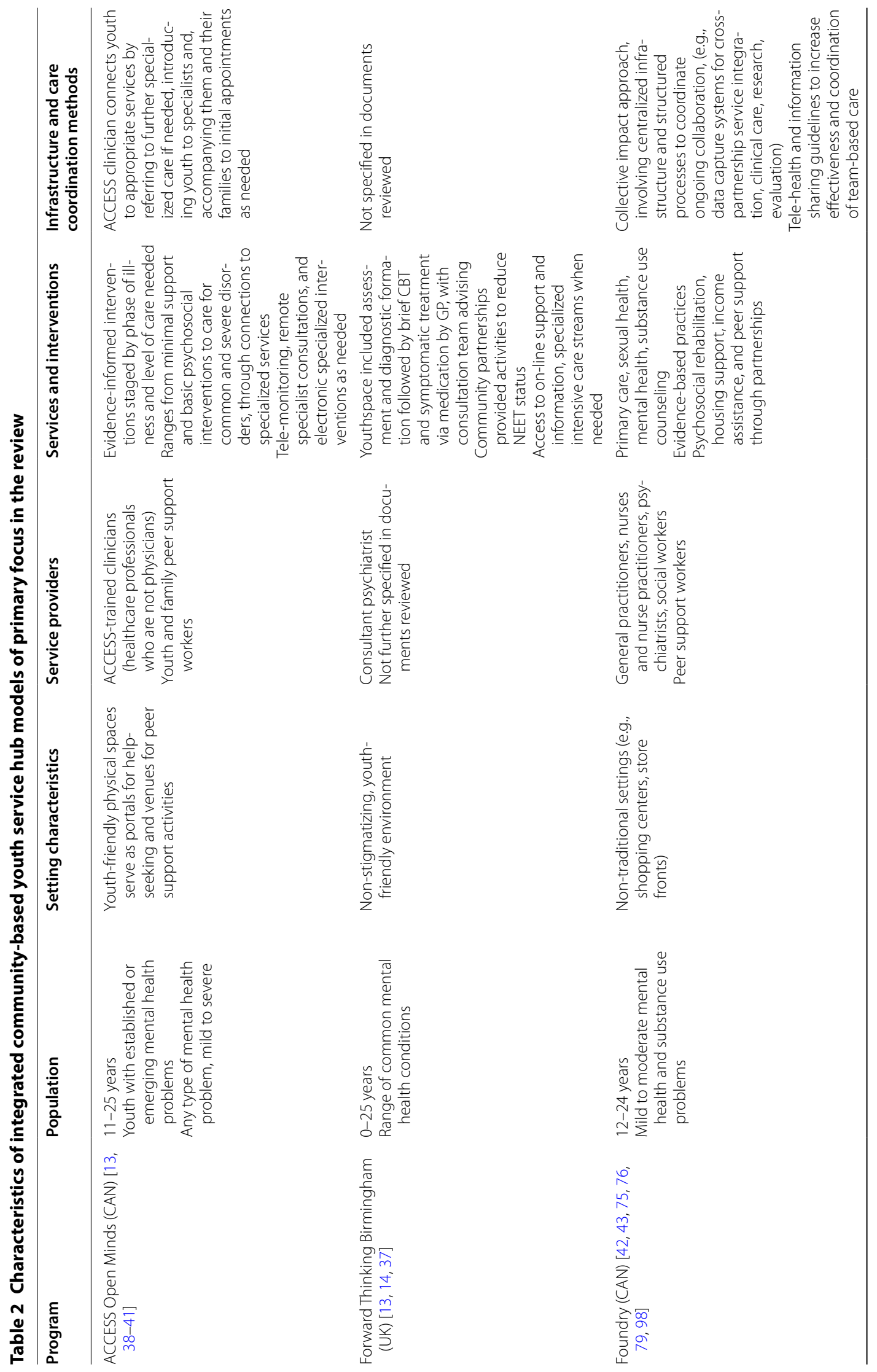












early adulthood. For example, headspace and Jigsaw include youth ages $12-25$ [30, 31, 36, 45], and ACCESS Open Minds serves youth ages 11-25 [38-41]. The 15-17 age range is the peak age of presentation at headspace [45-48] and Jigsaw [49], with an overrepresentation of females (e.g., headspace, 63.7\%) [45-48]. However, nearly half of youth at Jigsaw were male (43.5\%) [49]; there have been other encouraging findings regarding male youth accessing ICYSHs, despite evidence of greater difficulty engaging males in traditional mental health $(\mathrm{MH})$ treatment $[34,50]$. Service access at headspace is improved for youth who are socially and economically marginalized as compared to the general population, or those youth who lack support from their families and peers; however, young adults (18-25) and those from non-English speaking households are underrepresented [50].

Most models identified a focus on intervening during early stages of distress, possibly before diagnostic criteria are met $[13,36,39,40,43,51,52]$. In headspace centers, mood and anxiety symptoms and disorders were the most common presenting problem [46, 52-55], although formal diagnosis was available for only few clients [46]. Additionally, 69-73\% of headspace youth presented with high or very high levels of psychological distress [46, 48], and $19-29 \%$ of young adult clients were not engaged in education, employment or training $[46,56]$. Jigsaw similarly reported seeing youth with high levels of psychological distress [49], with four main types of issues identified among youth: developmental, comorbid, anxious and externalizing disorders [57].

\section{Settings}

Documents varied in the level of detail provided about service settings. Several documents emphasized efforts to make settings accessible to youth, non-stigmatizing, and youth-friendly. For example, some programs were located in shopping centers and storefronts and involved youth in decisions about design and décor $[43,58]$. Others were centrally located or close to public transportation [31, $38,50]$. In headspace centers, physical aspects of the settings described as youth-friendly include couches and bean bag chairs, colorful walls and creative artwork for a vibrant look, and an open waiting area and high ceilings to promote a feeling of safety and openness [59]. Youthfriendly environments were key for Foundry, including an informal, non-clinical space where youth can spend time with peers in addition to attending appointments [43]. Jigsaw discussed plans to develop a youth café with a public youth space and a program delivery area [34]. Some headspace centers encourage recreational use with pool and air-hockey tables [60].

\section{Service providers}

Documents describing the types of service providers typically include a range of professionals from several disciplines. For example, the intensive mobile youth outreach service subprogram of Orygen Youth Health consists of two psychologists, two social workers, one occupational therapist, one psychiatric nurse, and a part-time consultant psychiatrist [61]. Very few documents provided detailed information about service providers' roles. Within New Zealand's Youth One Stop Shops, several services are offered by physicians, nurses, counsellors, social workers and youth staff [62], providing primary care, sexual and reproductive health, mental health, drug and alcohol services, counselling, smoking cessation, family planning, and health promotion and education services [38]. For the Foundry prototype, primary care and sexual health services are provided by general practitioners and nurse practitioners, mental health services by psychiatrists, and substance use counseling by social workers and nurses. Additionally, partnerships provide other services, including psychosocial rehabilitation, housing support, income assistance, and peer support. ACCESS Open Minds clinicians include professionals with psychology, nursing, social work, or occupational therapy backgrounds who assess youth needs and provide need-based care $[40,43]$. In YouthCan IMPACT, each integrated collaborative care team includes youth workers, social workers, psychiatrists, nurse practitioners, peer support workers, access to primary care providers, and a care navigator [52]. For higher-risk youth, psychiatrists and/or nurse practitioners provide psychiatric assessment and medication management, along with other clinically appropriate interventions. Several ICYSH models in this review included peer support workers as service providers [38, 40, 43, 52, 63], reflecting the importance of youth with lived experience supporting other youth experiencing similar issues.

Many documents did not provide information on their service provider training process. Some documents broadly indicated that there is training in evidence-based interventions and best practices [36, 59, 64], in working with youth and youth-friendly approaches [59, 65], and professional development or continuing education activities $[38,66,67]$, though difficulties with releasing staff for professional development opportunities were also noted [38]. Some documents mentioned ongoing supervision and regular team meetings $[51,68]$. As part of Jigsaw, a structured training program addresses adolescent development and mental health, strengths-based and solution-focused approaches, risk assessment, goal setting, crisis response, interagency working and collaboration, 
consultation processes, and integrated planning and evaluation [34]. Initial training ensures a shared understanding of the Jigsaw philosophy and implementation process, with the plan of extending the training more broadly to others in the community who work with youth to increase service capacity.

\section{Services and interventions}

More information was provided on broad types of services included in ICYSHs than on specific interventions (Table 2). headspace includes four core service streams: mental health, drug and alcohol services, primary care, and vocational assistance (e.g., [31, 69, 70]). Primary care is a component of several other models (e.g., [38, 43, 71]). Social services, including vocational assistance, education and training support, and housing support were a component of other models [37,38]. Several models use brief, solution-focused interventions (e.g., [13, 34, 37, 45, $52]$ ), as well as peer support and mentoring (e.g., [34, 52, 72]).

Some models provided information on specific psychosocial interventions utilized. Services provided by Jigsaw include case management, problem-solving, mindfulness, cognitive-behavioral therapy (CBT), substance use services, psychoeducation, and social skills [57]. In headspace centers, CBT was reported to be the most common treatment provided for all presenting concerns, followed by supportive counseling and psychoeducation [73]. In YouthCan IMPACT, standardized protocols are used for interventions including solution-focused brief therapy (SFBT) and group dialectical behavioral therapy (DBT) skills [52]. Early intervention approaches for psychosis including CBT were mentioned as part of enhanced headspace services, ACCESS Open Minds, and Orygen Youth Health [13, 14, 41]. ACCESS Open Minds provides services depending on a youth's needs, including specific therapies (e.g. DBT for emotion dysregulation), transition to a specialist service (e.g. eating disorder program), or admission to a hospital (e.g. high risk of suicide) [40]. Some documents mentioned the use of evidence-based approaches, but not specific interventions (e.g., [42, 49]). For example, an independent headspace evaluation interim report indicated that implementation efforts were prioritized over tracking the extent of use of evidence-based treatments [74].

Intake and assessment processes were described in some papers; however, little information was provided on the use of standardized assessment measures. One exception, however, is the use of Kessler Psychological Distress Scale (K-10), Clinical Global Impression (CGI) scale, and the Social and Occupational Functioning Assessment Scale (SOFAS) by ACCESS Open Minds clinicians as part of a measurement-based care approach [40]. Some models mentioned following a stepped-care or clinical staging model (e.g., [51, 52]). Other work did not use this terminology, but described analogous processes. For example, headspace interventions are said to be delivered according to severity and complexity of an individual's needs [64]. Some documents discussed personalized treatment plans [37] and person-centered approaches [42].

\section{Infrastructure and care coordination}

Infrastructure aspects included use of health registries, outcome tracking, information sharing through electronic records, and cross-organization administrative processes, although many documents did not discuss infrastructure.

The Foundry model uses a centralized infrastructure and structured processes that coordinate ongoing collaboration, such as data capture systems that support crosspartnership service integration, clinical care, research, and evaluation [43]. Tele-health and information sharing guidelines are promoted to increase the effectiveness and coordination of team-based care $[75,76]$. At headspace, data are collected through a minimum dataset process in which all youth accessing centers and their service providers enter data electronically at each service occasion $[31,46]$. The headspace national office is responsible for infrastructure efforts including developing funding and assessment guidelines, negotiating contracts, developing reporting structures and key performance indicators, and providing policies and tools including partnership documentation, memorandums of understanding, governance guidelines and a business model guide [74]. In Jigsaw, youth data are captured through an online system that records clinical, case management, service delivery, and outcome-related information [49]. The use of a single, shared health record to facilitate collaboration and streamline care was mentioned in several documents (e.g., [58, 66, 72]). Shared infrastructure has been identified as positively contributing to service coordination and integration [74].

Other methods of care coordination include identifying point persons to facilitate service access and coordinate care across specialists, with titles such as care navigators, youth workers, and youth access clinicians [38, 51, 52, 77]. These workers engage in activities such as exchanging information and participating in joint coordination meetings and planning activities [74]. Some models discussed the role of support or outreach workers in linking clients to services outside the ICYSH and maintaining connection and follow up [34, 72]. Coordinating external referral pathways was viewed as helpful by youth accessing headspace services [59] and care coordination is a key headspace performance indicator [70]. 


\section{Common principles of integrated care models}

Several common principles of ICYSH models were discussed, including improving access to care and early intervention, youth and family engagement and participation, youth-friendly settings and services, evidenceinformed approaches, and partnerships and collaboration (Table 3).

\section{Improving access to care and early intervention}

A number of programs emphasized timely access to care, with the ACCESS Open Minds providing access to assessment within $72 \mathrm{~h}$ and appropriate care within 30 days [40, 41], the Forward Thinking Birmingham Program providing rapid response and assessment within 1 week [13, 37], and the Orygen Youth Health program providing a 24/7 triage, assessment and crisis response [14]. In addition, diverse access pathways were provided, e.g., self-referral [14, 31], walk-in or drop-in services [4144], and online access to services [48] including telemedicine $[41,42]$. Similarly, a number of programs highlighted the importance of early intervention [14, 31, 34, 36, 39, $43,52,78]$ and some included clinical staging [78] or stepped-care [52] to provide services at the earliest stage necessary.

\section{Youth and family engagement}

Multiple programs mentioned involving youth and families in design, implementation and evaluation of ICYSH models [14, 31, 34, 37-40, 43, 52, 57, 61, 74, 79, 80]. The programs established Youth Advisory Groups [31, 34, 43, $59,74]$ or conducted focus groups $[65,70,75]$ and surveys [70], and collected feedback from social media/websites [70] to ensure youth and family engagement.

\section{Youth-friendly settings and services}

Several documents mention establishing a non-stigmatizing setting $[37,43]$ with a youth-friendly environment $[13,31,37,38,40,43,52,70]$, for example with art [31, 70], couches, music, or recreational activities [38]. Youthfriendly services include not only the environment, but also welcoming and friendly staff $[31,38,42,60]$. headspace and Jigsaw engaged youth in the development of service settings in order to create a youth-friendly environment [31, 36, 74].

\section{Evidence-informed approaches}

All programs were found to stress the utilization of evidence-based practices [14, 31, 36, 39, 42, 52, 61, 65, 78], using CBT [14, 52, 65, 78], DBT [52], or other evidencebased or -informed practices [36, 42, 52, 61]. However, details regarding establishing and monitoring fidelity were rarely provided.

\section{Partnerships and collaborations}

Another core value was forming partnerships and collaborations with a diverse set of agencies. Most programs had partnerships with various stakeholders including youth, families, service providers, community agencies, researchers, hospitals, policy makers, etc. $[13,14,31$, $41,43,52]$. Partnerships help provide multidisciplinary, co-located services [38] including social, educational, employment services [14], housing support, income assistance [43] and other specialist services [78]. A social network analysis of the headspace staff reported interprofessional collaboration existed in both routine and uncertain situations [81]. In addition, a study conducted with internal and external staff of Youth One Stop Shops reported that individual staff's trusting personal relationships was crucial for successfully overcoming agency differences, and collaborative care was found to be essential to the successful care of youth with high needs [82].

\section{Outcome research and program evaluation}

Overall, research on youth $\mathrm{MH}$ or functional outcomes following intervention was limited, with only 11 documents reporting outcomes (Table 4). One additional article reported youth outcomes; however, the focus was on evaluating an intensive mobile outreach subcomponent rather than overall integrated care [83]. Outcomes reported were predominantly short-term, with long-term follow-up data lacking. Only one evaluation (non-RCT) included a comparison group [84], and few studies provided comparative outcome data to contextualize the magnitude of reported outcomes (exceptions: [51, 73]). Methods of measuring outcomes varied; however, several studies reported using standardized self-report and clinician-rated measures [45, 51, 73, 83-86]. Three documents provided some outcomes information, but did not specify the measures used [37, 38, 87]. In addition to the 11 documents with youth outcome information, three articles reported on case study findings; of them, one utilized a standardized self-report measure [65], whereas two did not [61, 78]. Seven documents discussed future outcome measurement plans, spanning a total of three ICYSH models, all in Canada [13, 39, 41, 43, 44, 52, 88]. Generally, positive outcomes, particularly improvements in psychological distress and psychosocial functioning have been found [37, 45, 73, 84]. Additional evaluation projects are under way by ACCESS Open Minds using a stepped-wedge design $[39,40]$ and YouthCan IMPACT using a pragmatic randomized controlled trial [52]. ACCESS Open Minds and the Foundry also plan to 


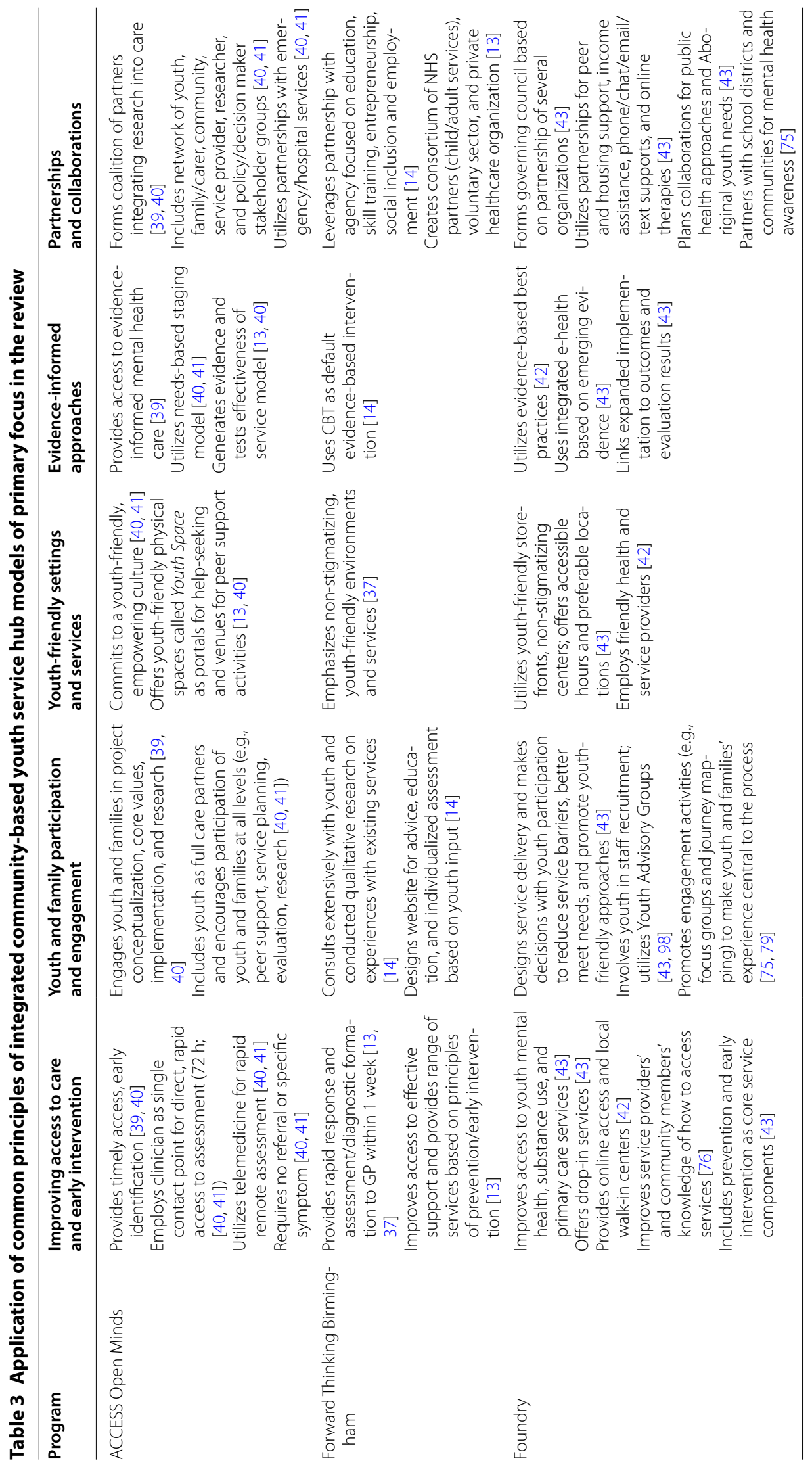



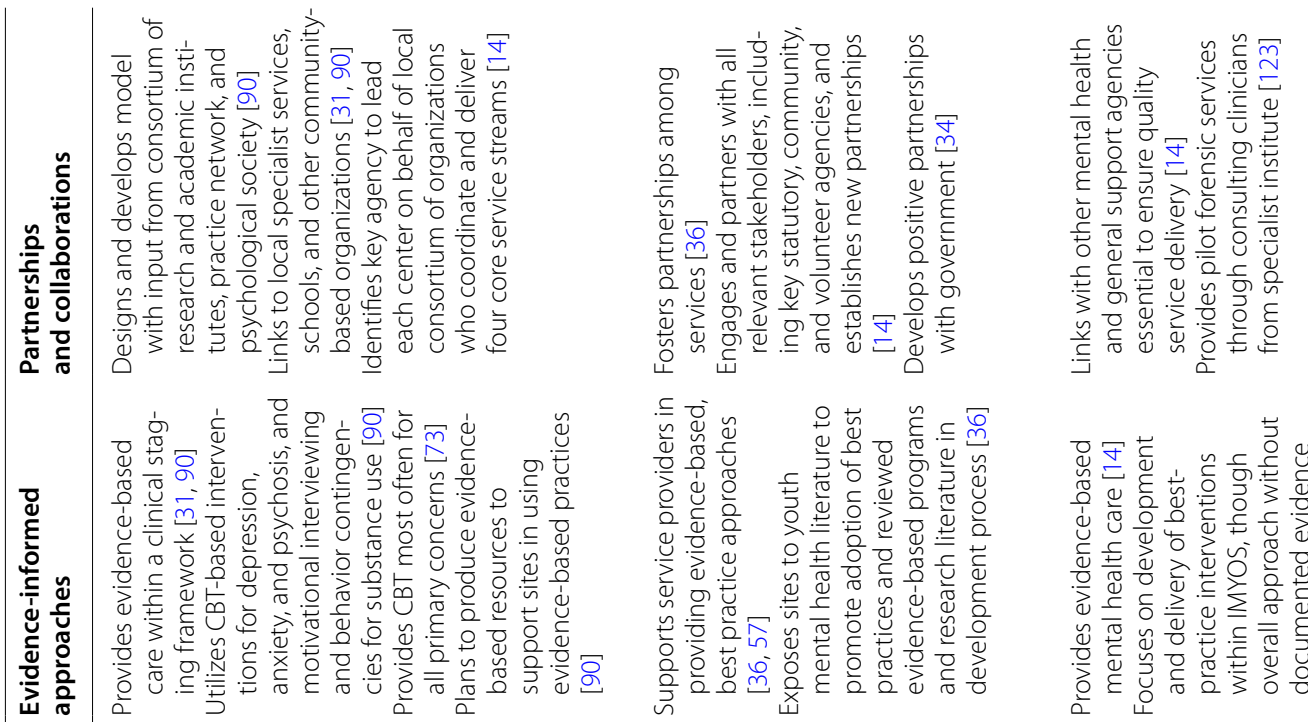

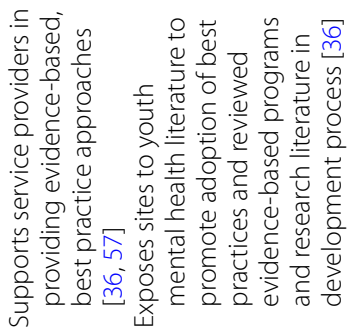



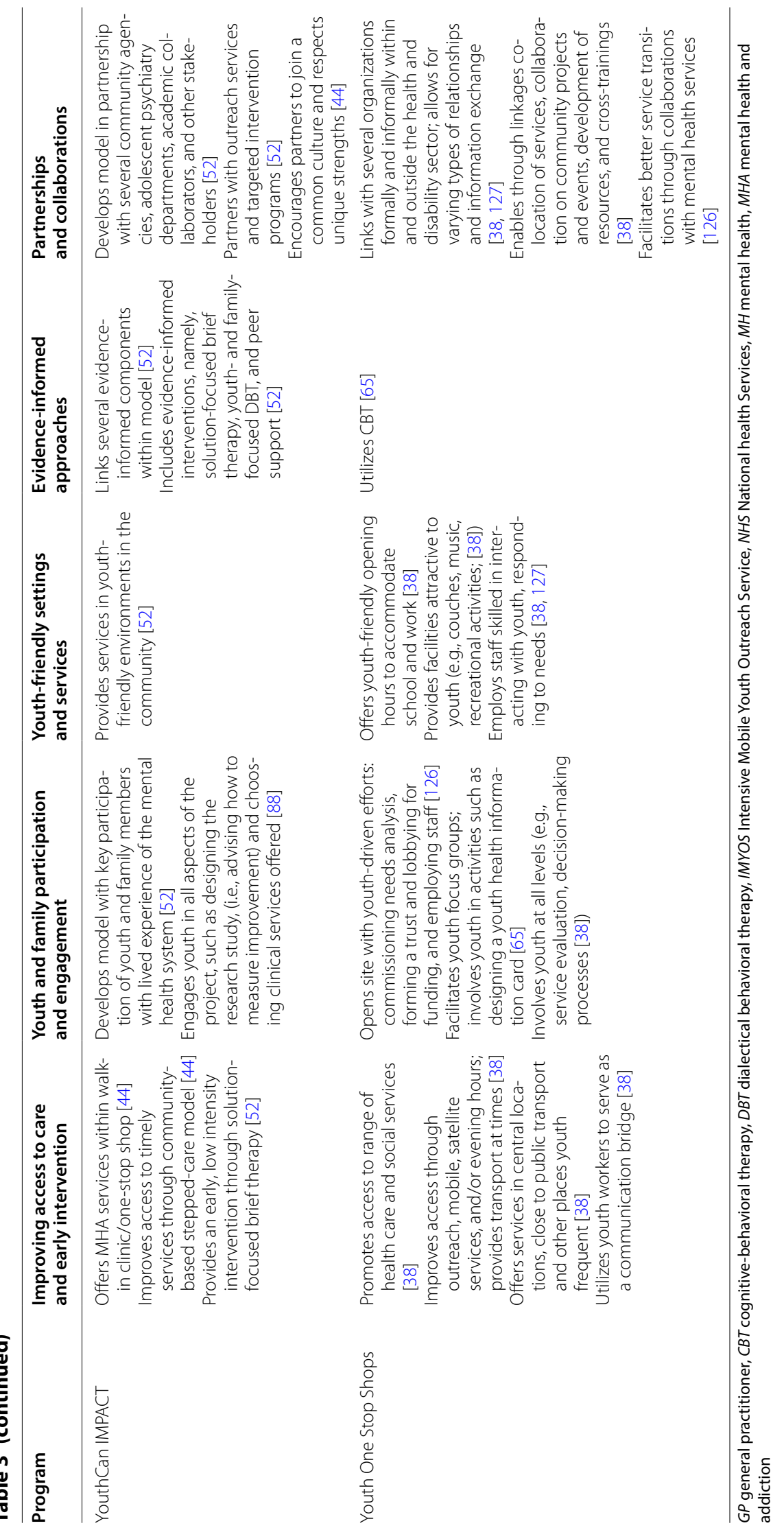




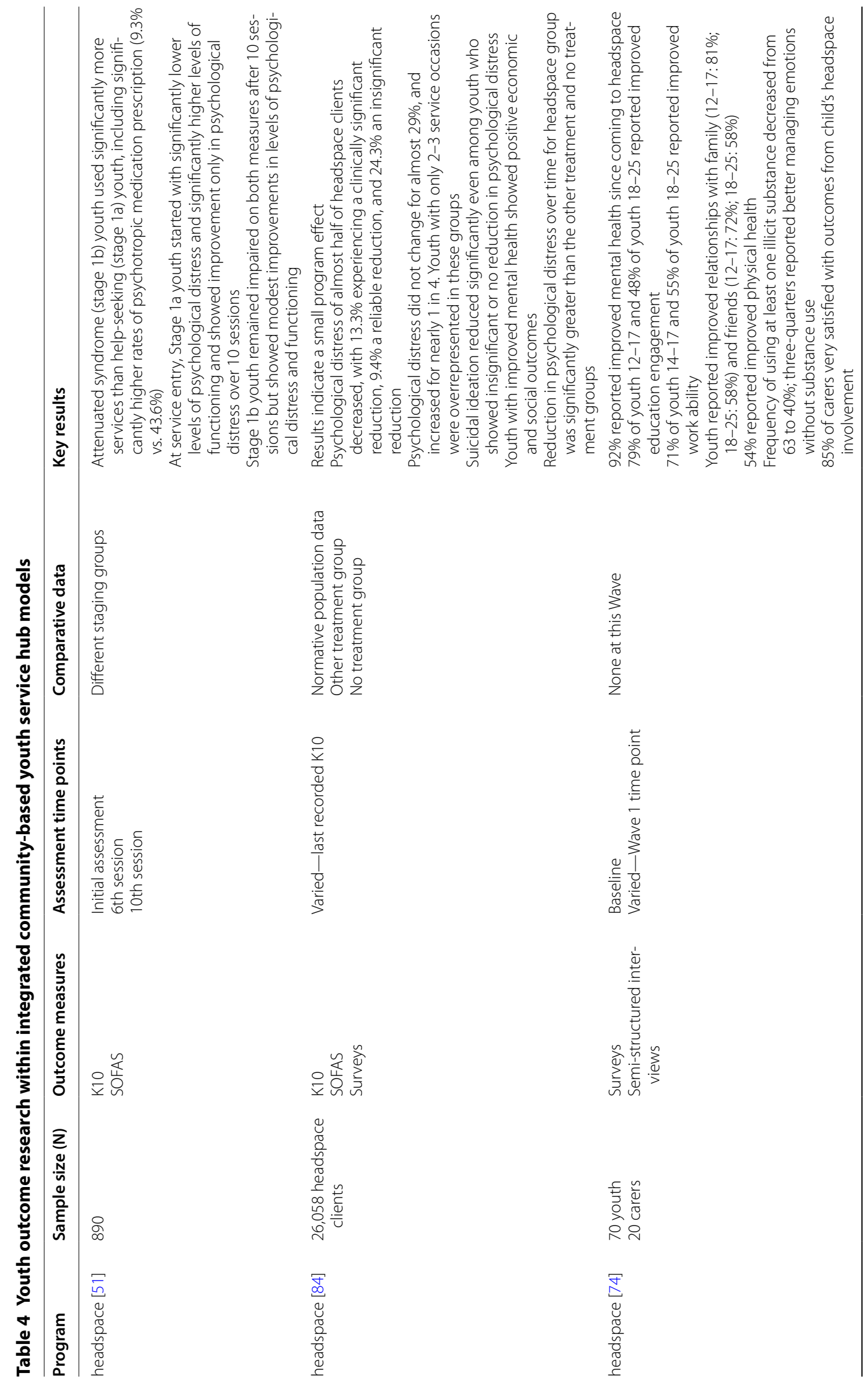




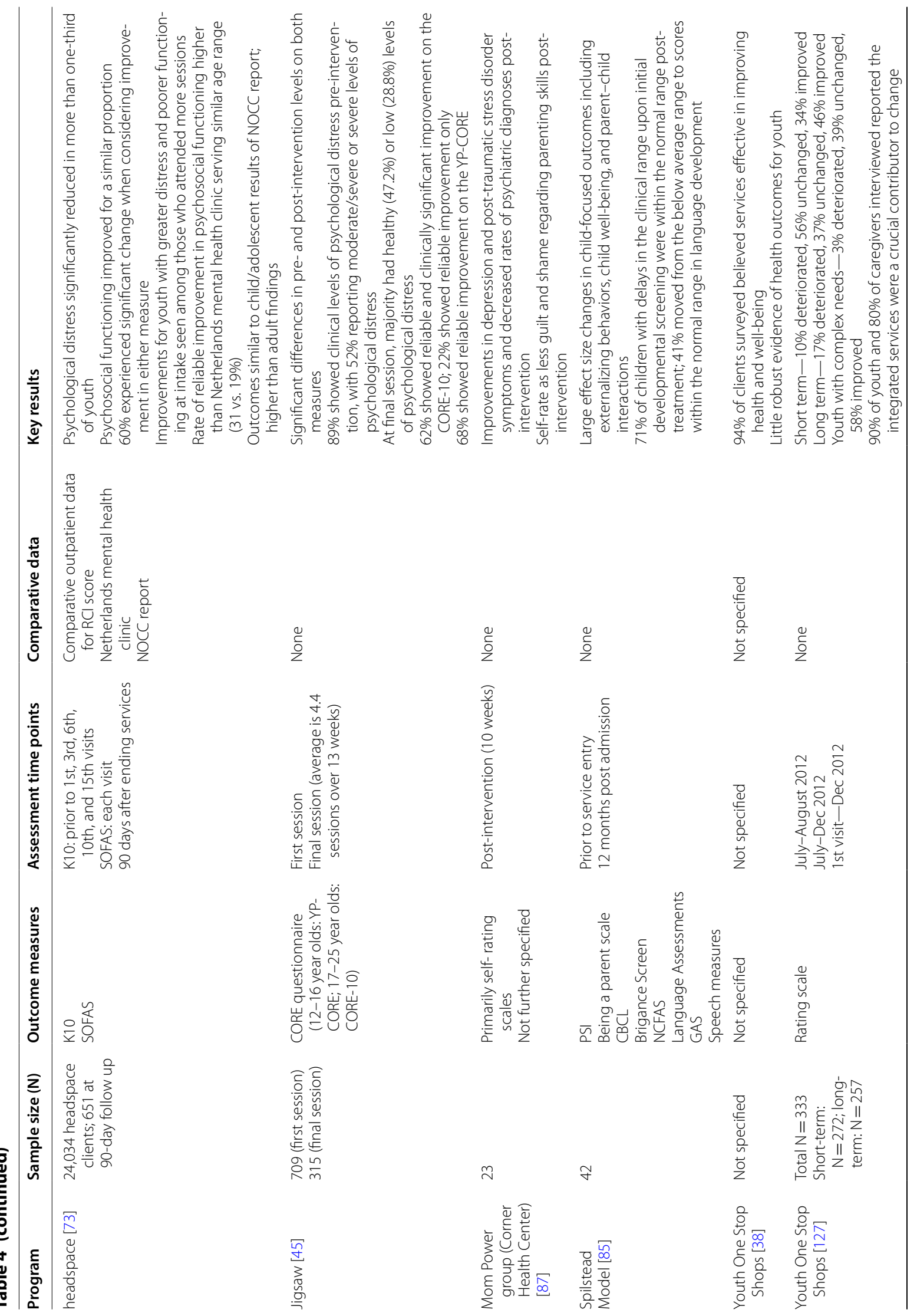







test the effectiveness of the individual interventions being used [13, 43], adding to the intervention evidence base.

\section{Discussion}

This scoping review identified international ICYSH models for addressing youth $\mathrm{MH}$ needs. Common principles and characteristics of these models are summarized. This review provides a synthesis of the components of such models and can serve as a foundation for informing replication, evaluation and implementation of ICYSHs, which have been rapidly proliferating $[13,14]$. However, limited information was provided in many domains, raising questions about the availability of sufficient detail to achieve these goals.

ICYSH models showed some similar characteristics, providing a starting point for replication. Many have focused on early intervention for adolescents and young adults [14, 34, 36, 39, 43, 52, 78], and on addressing the unmet need for services across the transition to adulthood [12]. Nearly all models reported trying to develop non-stigmatizing, youth-friendly environments [89]. Reported efforts were consistent with recommended approaches for improving accessibility and youth-friendliness laid out within calls for youth mental health services reform [90]. Little information was available on service providers' training or specific roles. More information was provided on broad services categories, with mental health, drug and alcohol services, primary care, vocational or other social services, and peer support featured in several models. Evidence-based and brief, solution-focused interventions were promoted, but specific interventions, extent of their use and achievement of fidelity were rarely reported. Some information was identified on care coordination and infrastructure, such as administrative processes, information sharing, and outcome tracking, although many documents did not discuss these aspects. Overall, the variability in the level of detail provided for several of the characteristics examined precludes drawing strong conclusions regarding the extent of differences across models, and does not allow for clear direction regarding replication, evaluation of these components, nor wide scale implementation. Future reporting should follow TIDieR guidelines in order to facilitate replication, evaluation and implementation [91].

However, there was clearer consistency in the aims of ICYSH models. Improved access to care and earlier intervention were frequently highlighted and served as catalysts for model development. Engaging youth and families in service delivery and design were universally evident. Youth-friendly services and environments were emphasized within all models. Disseminating evidenceinformed interventions to more youth in need and implementing evidence-based care were also consistently identified. Finally, leveraging partnerships and collaborations to provide multidisciplinary, integrated services or community capacity building was common. Details regarding the 'how' of effectively achieving these aims, however, were limited, thus falling short of providing the information required for replication, evaluation and implementation-related decision-making. For example, the literature is lacking sufficient information on the formation and maintenance of partnerships and related agreements, decision-making processes, and fidelity to evidence-based interventions. Moreover, although integration was frequently mentioned as an aim, there was little explanation of how services are integrated on a structural level beyond the role of care coordinators, and measurement of service integration was lacking.

Notably, the review also revealed potential limitations of these models of care. Specifically, limited service availability [33] and workforce shortages [92] are challenges for the field more broadly and also impact ICYSHs. Additionally, several studies suggest that at least some of the youth presenting for services were experiencing more distress and impairment than models may have been primarily designed to address $[49,51,59,93]$, which is a key finding that should be taken into account in the design and development of robust ICYSH models that can meet the needs of all presenting youth. The complexity of managing numerous objectives and components within a comprehensive ICYSH may limit the ability to fully address each aspect simultaneously.

This review also identified a number of other limitations of the literature. Notably, rigorous evidence of improved youth outcomes attributable to these models of care is scarce. The limited outcome research identified was short-term and lacked control groups (see [19] for a thorough review). All models are from high-income countries, limiting generalizability. Also important to note is the limited translating of work into publications; for some models, the bulk of information was derived from grey literature sources. Finally, numerous relevant documents were not captured using integration-focused search terms (see Appendix), raising questions about terminology consistency.

\section{Implications for research}

This review highlights the pressing need for more rigorous research examining youth outcomes within ICYSHs. Notably, robust designs are needed to evaluate both the effect of ICYSHs on youth outcomes and cost-effectiveness compared to usual treatment. Such efforts are underway for the YouthCan IMPACT program, which is comparing ICYSHs to hospital-based adolescent psychiatric services [52]. ACCESS Open Minds includes a cluster RCT design to evaluate implementation processes, 
but not youth health outcomes [41]. Additional comparative effectiveness trials comparing treatment outcomes within different ICYSH models, utilizing different control interventions, would strengthen the literature (see also [19]). Given that much information was derived from grey literature, findings also suggest the need to translate more of this work into peer-reviewed publications to facilitate replication and rigorous evaluation. Publications regarding ICYSHs need to include greater detail regarding key attributes, especially those considered unique to the model, such as integration of care, partnership agreements, integrated decision-making processes and youth friendliness to allow for replicability [94]. In addition, supplementary material could be provided on project websites to more thoroughly describe models in a level of detail often not possible within the framework of academic publications. Given the lack of detail on certain aspects of these models, there is insufficient information currently available in these areas to guide implementation.

Utilizing common language and terminology regarding service integration would also improve information sharing and replicability. Reaching a consensus on the most essential elements of these models should be prioritized, for example the types of early interventions that should to be included, but also other characteristics such as types of service providers and other site features. Findings from this review will inform the appraisal of key features of such models, as does the recent review by Hetrick et al. [19]. Additionally, research within established programs could address challenges related to delivering and tracking the use of evidence-based practices in community settings. Finally, using common, core outcome measures [95] to track outcomes and other aspects of ICYSHs would help facilitate collaborative research efforts, support future meta-analyses, and more rapidly advance the field.

\section{Implications for practice}

The finding that youth are presenting to ICYSHs with high levels of distress and impairment-some with more serious needs than models were designed for-has implications for service availability, accessibility, resource allocation, and training. If ICYSH models become a default "stop gap" for youth with more serious difficulties due to a lack of appropriate services elsewhere, their ability to address the needs of youth with emerging $\mathrm{MH}$ concerns may be limited. Having clear processes in place supporting a no-wrong-door approach and linking youth with the most appropriate services could lessen these concerns. Leveraging existing service relationships and creating new linkages may be necessary to ensure that youth receive appropriate, comprehensive, multidisciplinary services. These linkages may cross academic-community divides, which may require new ways of working collaboratively (e.g., medical vs. community mental health model). Finally, defining a core basket of services for ICYSH models, as is being discussed in the broader mental health services field (e.g., [96]), will also be important for clarifying the needs that can effectively be met and ensuring that service providers receive appropriate specialized training.

\section{Implications for policy}

This literature synthesis can guide policymakers interested in transforming the youth mental health service system through ICYSH implementation. Policymakers should be aware of the identified deficits in the mental health service system driving the development of ICYSH models and their potential to address these pressing concerns. Policy planning, communication, and funding complexities should be taken into account since comprehensive, cross-sectoral services may span multiple government departments (e.g., youth services, health services, education). Policymakers should be aware that creating ICYSH models does not necessarily require building new services, but can be accomplished by integrating existing services and leveraging existing partnerships, which is encouraging for financial feasibility.

Although much remains to be determined, particularly with regard to outcomes, the profiles of youth best served by such models, cost-effectiveness, and optimal components, this review provides a starting point for understanding the common characteristics of ICYSH models that are likely to be important targets for replication, evaluation, service development efforts and funding. Research granting agencies are encouraged to support rigorous evaluations of ICYSH models to further inform development. Results from economic analyses (e.g., [41, 52]) will have particularly important implications for policy decisions. Policymakers are encouraged to continue to monitor this burgeoning field for enhanced guidance as they develop and expand ICYSHs internationally.

\section{Limitations}

There are limitations of this review that warrant consideration. Although efforts were made to ensure that the review is as thorough and comprehensive as possible, there is inevitably unidentified relevant literature. In particular, the grey literature search was representative, not exhaustive. Additionally, this review was limited to English publications. Documents were found to originate from high-income countries. This may affect the generalizability of the findings to low- or middle-income countries. Information was generally not available on funding models supporting the various ICYSH initiatives; this 
should be further explored to support new implementation initiatives. To allow for in-depth examination of ICYSHs, this review did not include literature on integrating behavioral health services into existing primary care or school settings, although there may be relevant findings from these areas. Aspects of primary care or school-based models should also be considered in future research to determine the ideal setting. Finally, in accordance with scoping review methodology [21], the quality of studies included in this review was not assessed.

\section{Conclusions}

This review brings together literature on ICYSH models for youth $\mathrm{MH}$ from diverse sources and examines their common principles and characteristics. ICYSHs are poised to address many of the most pressing youth mental health service concerns by uniting traditionally fragmented services into single, youth-friendly settings, improving early access to evidence-informed interventions, engaging youth and families, and drawing on the strengths of cross-disciplinary and multi-sectorial collaborations. Future efforts to provide comprehensive descriptions and replicate these models, evaluate youth outcomes and identify the most critical components and processes for ICYSHs will further strengthen these models of care.

\section{Acknowledgements \\ The authors thank the McCain Centre Youth Engagement Team for their support. They would also like to acknowledge staff members Sarah Oates, Navitha Jayakumar, and Alisha Williams for their support in the document selection process. Fiona Inglis' contribution to the database search is also acknowledged.}

\section{Authors' contributions}

$\mathrm{CS}, \mathrm{LH}, \mathrm{KC}, \mathrm{GC}, \mathrm{AC}, \mathrm{MR}$, PS and JH contributed to the concept and design of the review. MR drafted the search strategy and conducted the literature search. CS drafted the protocol and all authors contributed to the revision and finalization of the manuscript. JH is the guarantor of this work. All authors read and approved the final manuscript.

\section{Funding}

This review was conducted with the financial support of the Ontario SPOR SUPPORT Unit (OSSU), which is supported by the Canadian Institutes of Health Research and the Province of Ontario. It was also made possible through a financial contribution from the Margaret and Wallace McCain Centre for Child, Youth and Family Mental Health, Centre for Addiction and Mental Health $(\mathrm{CAMH})$. The funders play no role in the design of the review or the collection, analysis, and interpretation of the data.

\section{Availability of data and materials}

The search strategy is available as an Appendix.

\section{Ethics approval and consent to participate}

As a scoping review of the literature, research ethics board approval was not required for this study.

\section{Consent for publication}

Not applicable.

\section{Competing interests}

The authors declare that they have no competing interests.

\section{Author details}

${ }^{1}$ Margaret and Wallace McCain Centre for Child, Youth and Family Mental Health, Centre for Addiction and Mental Health, Toronto, ON, Canada.

${ }^{2}$ Lawrence S. Bloomberg Faculty of Nursing, University of Toronto, Toronto, ON, Canada. ${ }^{3}$ Department of Psychiatry, University of Toronto, Toronto, ON, Canada. ${ }^{4}$ Hurvitz Brain Sciences Research Program, Sunnybrook Research Institute, Toronto, ON, Canada. ${ }^{5}$ McMaster University, Hamilton, ON, Canada.

${ }^{6}$ Department of Psychiatry, Hospital for Sick Children, Toronto, ON, Canada.

\section{Appendix: Search strategy: Medline-OVID}

1. Delivery of Health Care, Integrated/ (10004)

2. (integrat* adj2 (care or treatment* or therapy or team* or services)).kf,ti.

3. (integrat" adj2 (care or treatment* or therapy or team* or services)).ab. /freq $=2$

4. (shared adj2 (care or treatment* or therapy or team* or services)).ti,kf.

5. (multidisciplinary adj2 (care or treatment" or therapy or team* or services)).ti,kf.

6. (multidisciplinary adj2 (care or treatment" or therapy or team* or services)).ab. /freq $=2$

7. (shared adj2 (care or treatment* or therapy or team" or services) ).ab. /freq $=2$

8. (shared adj2 (care or treatment* or therapy or team* or services)).ti,kf.

9. (multi-disciplinary adj2 (care or treatment" or therapy or team* or services)).ti,kf.

10. (multi-disciplinary adj2 (care or treatment" or therapy or team" or services)).ab. /freq $=2$

11. (coordinat" adj2 (care or treatment" or therapy or team" or services)).ti,kf.

12. (coordinat" adj2 (care or treatment* or therapy or team* or services)).ab. /freq $=2$

13. (co-ordinat" adj2 (care or treatment" or therapy or team" or services)).ti,kf.

14. (co-ordinat" adj2 (care or treatment* or therapy or team" or services) $)$.ab. /freq $=2$

15. (multiagency adj2 (care or treatment" or therapy or team" or services)).ti,kf.

16. (multiagency adj2 (care or treatment* or therapy or team* or services)).ab. /freq $=2$

17. (joint working adj2 (care or treatment* or therapy or team" or services)).ti,kf.

18. (joint working adj2 (care or treatment" or therapy or team* or services)).ab. /freq $=2$

19. one stop shop.tw.

20. system of care.ti,kf.

21. system of care.ab. / freq $=2$

22. 1 or 2 or 3 or 4 or 5 or 6 or 7 or 8 or 9 or 10 or 11 or 12 or 13 or 14 or 15 or 16 or 17 or 18 or 19 or 20 or 21

23. Mental Health/

24. mental health.ti,kf. 
25. exp Mental Health Services/

26. exp mental disorders/

27. ((behavio? $\mathrm{r}^{*}$ or mental) adj2 (health or disorder* or problem*)).ti,kf.

28. psychiatry/ or adolescent psychiatry/ or child psychiatry/ or psychology, child/ or psychology, developmental/

29. ((obsessive compulsive or panic or post traumatic stress or eating or bipolar or oppositional defiant or conduct) adj2 disorder*).ti,kf,kw.

30. (depress* or anxiety or substance abuse or drug abuse or alcoholism or alcohol abuse or disruptive behavio?r disorder* or attention deficit or autism or asperger*).ti,kf,kw.

31. (agoraphobia or PTSD or trauma or OCD or anorexia or bulimia or psychosis).ti,kf,kw.

32. or $/ 23-31$

33. 22 and 32

34. (pediatric* or paediatric ${ }^{*}$ or child* or adolescent? or youth? or teenag* or teen? or boys or girls or young adult? or emerging adult?).ti,kf,ab,jn.

35. 33 and 34

36. limit 33 to ("all child ( 0 to 18 years)" or "child (6 to 12 years)" or "adolescent (13 to 18 years)" or "young adult (19 to 24 years)")

37. 35 or 36

38. limit 37 to english language

39. limit 38 to $y r=$ "2001 -Current"

40. limit 39 to (comment or editorial or letter)

41. 39 not 40

Received: 24 October 2018 Accepted: 10 July 2019

Published online: 23 July 2019

\section{References}

1. Kessler RC, Berglund P, Demler O, Jin R, Merikangas KR, Walters EE. Lifetime prevalence and age-of-onset distributions of DSM-IV disorders in the national comorbidity survey replication. Arch Gen Psychiatry. 2005;62(6):593-602

2. Kim-Cohen J, Caspi A, Moffitt TE, Harrington H, Milne BJ, Poulton R. Prior juvenile diagnoses in adults with mental disorder: developmental follow-back of a prospective-longitudinal cohort. Arch Gen Psychiatry. 2003;60(7):709-17.

3. Kieling C, Baker-Henningham H, Belfer M, Conti G, Ertem I, Omigbodun $\mathrm{O}$, et al. Child and adolescent mental health worldwide: evidence for action. Lancet. 2011;378(9801):1515-25.

4. Polanczyk GV, Salum GA, Sugaya LS, Caye A, Rohde LA. Annual research review: a meta-analysis of the worldwide prevalence of mental disorders in children and adolescents. J Child Psychol Psychiatry. 2015;56(3):345-65.

5. Merikangas KR, He J-P, Burstein M, Swanson SA, Avenevoli S, Cui L, et al. Lifetime prevalence of mental disorders in U.S. adolescents: results from the National Comorbidity Survey-Adolescent supplement (NCS-A). J Am Acad Child Adolesc Psychiatry. 2010;49(10):980-9.

6. Ormel J, Raven D, van Oort F, Hartman CA, Reijneveld SA, Veenstra $R$, et al. Mental health in Dutch adolescents: a TRAILS report on prevalence, severity, age of onset, continuity and co-morbidity of DSM disorders. Psychol Med. 2014;45(2):345-60.

7. Ratnasingham S, Cairney J, Manson H, Rehm J, Lin E, Kurdyak P. The burden of mental illness and addiction in Ontario. Can J Psychiatry. 2013;58(9):529-37.

8. Whiteford HA, Degenhardt L, Rehm J, Baxter AJ, Ferrari AJ, Erskine HE, et al. Global burden of disease attributable to mental and substance use disorders: findings from the Global Burden of Disease Study 2010. Lancet. 2013;382(9904):1575-86.

9. Wang PS, Angermeyer M, Borges G, Bruffaerts R, Tat Chiu WAI, De Girolamo $\mathrm{G}$, et al. Delay and failure in treatment seeking after first onset of mental disorders in the World Health Organization's World Mental Health Survey Initiative. World Psychiatry. 2007;6(3):177-85.

10. Costello J, He J, Sampson N, Kessler R, Merikangas K. Services for adolescents with psychiatric disorders: 12-month data from the National Comorbidity Survey-Adolescent. Psychiatr Serv. 2014;65(3):359-66.

11. de Voursney D, Huang LN. Meeting the mental health needs of children and youth through integrated care: a systems and policy perspective. Psychol Serv. 2016;13(1):77-91.

12. McGorry PD. The specialist youth mental health model: strengthening the weakest link in the public mental health system. Med J Aust. 2007;187(7):S53.

13. Malla A, lyer S, McGorry PD, Cannon M, Coughlan H, Singh S, et al. From early intervention in psychosis to youth mental health reform: a review of the evolution and transformation of mental health services for young people. Soc Psychiatry Psychiatr Epidemiol. 2016;51(3):319-26.

14. McGorry PD, Bates T, Birchwood M. Designing youth mental health services for the 21st century: examples from Australia, Ireland and the UK. Br J Psychiatry. 2013;54:s30-5.

15. Ontario Ministry of Health and Long Term Care. Ontario providing faster access to mental health services for thousands of people. Toronto: Ontario Ministry of Health and Long-Term Care; 2017.

16. Kolko DJ, Perrin E. The integration of behavioral health interventions in children's health care: services, science, and suggestions. J Clin Child Adolesc Psychol. 2014;43(2):216-28.

17. Njoroge WF, Hostutler CA, Schwartz BS, Mautone JA. Integrated behavioral health in pediatric primary care. Curr Psychiatry Rep. 2016;18(12):106.

18. Asarnow JR, Rozenman M, Wiblin J, Zeltzer L. Integrated medical-behavioral care compared with usual primary care for child and adolescent behavioral health: a meta-analysis. JAMA Pediatr. 2015;169(10):929-37.

19. Hetrick SE, Bailey AP, Smith KE, Malla A, Mathias S, Singh SP, et al. Integrated (one-stop shop) youth health care: best available evidence and future directions. Med J Aust. 2017;207(10):S5-s18.

20. Canadian Institutes of Health Research. A guide to knowledge synthesis. 2010. http://www.cihr-irsc.gc.ca/e/41382.html. Accessed 21 Sept 2018.

21. Arksey H, O'Malley L. Scoping studies: towards a methodological framework. Int J Soc Res Methodol. 2005;8(1):19-32.

22. Levac D, Colquhoun H, O'Brien KK. Scoping studies: advancing the methodology. Implement Sci. 2010;5(1):69.

23. O'Brien KK, Colquhoun H, Levac D, Baxter L, Tricco AC, Straus S, et al. Advancing scoping study methodology: a web-based survey and consultation of perceptions on terminology, definition and methodological steps. BMC Health Serv Res. 2016;16(1):305.

24. Settipani C, Cleverley K, Hawke LD, Rice M, Henderson J. Essential components of integrated care for youth with mental health and addiction needs: protocol for a scoping review. BMJ Open. 2017;7(4):1-5.

25. Canadian Agency for Drugs and Technology in Health. Grey matters: a practical search tool for evidence-based medicine. CADTH Information Services; 2015.

26. United Nations Department of Economic and Social Affairs. Definition of youth. 2013.

27. Gröne O, Garcia-Barbero M. Integrated care. Int J Integr Care. 2001;1(e21):1-10.

28. Kodner DL, Spreeuwenberg C. Integrated care: meaning, logic, applications, and implications - a discussion paper. Int J Integr Care. 2002;2(4):1-6.

29. Delman J. Participatory action research and young adults with psychiatric disabilities. Psychiatr Rehabil J. 2012;35(3):231-4. 
30. McGorry PD, Tanti C, Stokes R, Hickie IB, Carnell K, Littlefield LK, et al. headspace: Australia's National Youth Mental Health Foundationwhere young minds come first. Med J Aust. 2007;187(7):S68-70.

31. Rickwood D, Paraskakis M, Quin D, Hobbs N, Ryall V, Trethowan J, et al. Australia's innovation in youth mental health care: the headspace centre model. Early Interv Psychiatry. 2019;13(1):159-66.

32. McGorry P, Trethowan J, Rickwood D. Creating headspace for integrated youth mental health care. World Psychiatry. 2019;18(2):140-1.

33. Cosgrave EM, Yung AR, Killackey EJ, Buckby JA, Godfrey KA, Stanford CA, et al. Met and unmet need in youth mental health. J Ment Health. 2008;17(6):618-28.

34. Bates T, Illback RJ, Scanlan F, Carroll L. Somewhere to turn to, someone to talk to. Headstrong - The National Centre for Youth Mental Health. Dublin: Headstrong; 2009.

35. Donnelly A, O'Reilly A, Dolphin L, O'Keeffe L, Moore J. Measuring the performance of the Mental Health Continuum-Short Form (MHC-SF) in a primary care youth mental health service. Ir J Psychol Med. 2019;. https://doi.org/10.1017/ipm.2018.55.

36. Illback RJ, Bates T, Hodges C, Galligan K, Smith P, Sanders D, et al. Jigsaw: engaging communities in the development and implementation of youth mental health services and supports in the Republic of Ireland. J Ment Health. 2010;19(5):422-35.

37. Vyas NS, Birchwood M, Singh SP. Youth services: meeting the mental health needs of adolescents. Ir J Psychol Med. 2014;32(01):13-9.

38. New Zealand Ministry of Health. Evaluation of youth one stop shops: synopsis report. 2009 .

39. ACCESS Open Minds. Project overview. 2017.

40. Malla A, lyer S, Shah J, Joober R, Boksa P, Lal S, et al. Canadian response to need for transformation of youth mental health services: ACCESS Open Minds (Esprits ouverts). Early Interv Psychiatry. 2019;13(3):697-706

41. Iyer SN, Boksa P, Lal S, Shah J, Marandola G, Jordan G, et al. Transforming youth mental health: a Canadian perspective. Ir J Psychol Med. 2015;32(01):51-60.

42. Foundry. Brand Story. 2016. https://foundrybc.ca/app/themes/foundry/ assets/docs/BCIYSI-Brand-Story.pdf. Accessed 21 Sept 2018.

43. British Columbia Integrated Youth Services Initiative (BC-IYSI). Rationale and Overview. 2015

44. Szatmari P, Henderson J, Cheung A, Cleverley K, Chaim G, Hawke L. Transforming youth mental health services in Canada: YouthCan IMPACT. World Child Adolesc Psychiatry. 2016;10:14-5.

45. O'Keeffe L, O'Reilly A, O'Brien G, Buckley R, Illback R. Description and outcome evaluation of Jigsaw: an emergent Irish mental health early intervention programme for young people. Ir J Psychol Med. 2015;32(01):71-7.

46. Rickwood D, Telford NR, Parker AG, Tanti CJ, McGorry PD. headspaceAustralia's innovation in youth mental health: who are the clients and why are they presenting? Med J Aust. 2014;200(2):108-11.

47. Bassilios B, Telford N, Rickwood D, Spittal MJ, Pirkis J. Complementary primary mental health programs for young people in Australia: access to Allied Psychological Services (ATAPS) and headspace. Int J Ment Health Syst. 2017;11(1):19.

48. Rickwood D, Webb M, Kennedy V, Telford N. Who are the young people choosing web-based mental health support? Findings from the implementation of Australia's national web-based youth mental health service, eheadspace. JMIR Ment Health. 2016;3(3):e40.

49. O'Reilly A, Illback R, Peiper N, O'Keeffe L, Clayton R. Youth engagement with an emerging Irish mental health early intervention programme (Jigsaw): participant characteristics and implications for service delivery. J Ment Health. 2015;24(5):283-8.

50. Patulny R, Muir K, Powell A, Flaxman S, Oprea I. Are we reaching them yet? Service access patterns among attendees at the headspace youth mental health initiative. Child Adolesc Ment Health. 2013;18(2):95-102.

51. Cross SP, Hermens DF, Hickie IB. Treatment patterns and short-term outcomes in an early intervention youth mental health service. Early Interv Psychiatry. 2016;10(1):88-97.

52. Henderson JL, Cheung A, Cleverley K, Chaim G, Moretti ME, de Oliveira $C$, et al. Integrated collaborative care teams to enhance service delivery to youth with mental health and substance use challenges: protocol for a pragmatic randomised controlled trial. BMJ Open. 2017;7(2):e014080.
53. Kwan B, Rickwood DJ, Telford NR. Development and validation of MyLifeTracker: a routine outcome measure for youth mental health. Psychol Res Behav Manag. 2018;11:67.

54. Rickwood DJ, Telford NR, Mazzer KR, Parker AG, Tanti CJ, McGorry PD. The services provided to young people through the headspace centres across Australia. Med J Aust. 2015;202(10):533-6.

55. Scott EM, Hermens DF, Glozier N, Naismith SL, Guastella AJ, Hickie IB. Targeted primary care-based mental health services for young Australians. Med J Aust. 2012;196(2):136-40.

56. O'Dea B, Glozier N, Purcell R, McGorry PD, Scott J, Feilds K-L, et al. A cross-sectional exploration of the clinical characteristics of disengaged (NEET) young people in primary mental healthcare. BMJ Open. 2014;4(12):e006378.

57. Peiper N, Illback RJ, O'Reilly A, Clayton R. Latent class analysis of need descriptors within an Irish youth mental health early intervention program toward a typology of need. Early Interv Psychiatry. 2017;11(1):37-46.

58. Callaly T, Dodd S, Ackerly C, Hantz P, O'Shea M, Berk M. Description and qualitative evaluation of Jigsaw, an integrated young persons' mental health program. Australas Psychiatry. 2009;17(6):480-3.

59. Rickwood D, Anile G, Telford N, Thomas K, Brown A, Parker A. Service Innovation Project Component 1: best practice framework. Melbourne: headspace National Youth Mental Health Foundation; 2014.

60. Medlow S, Kelk N, Cohen A, Hickie I. Facilitating early intervention: experiences of young people and implications for the shaping of headspace services. Australas Psychiatry. 2010;18(4):335-9.

61. Ryall V, Radovini S, Crothers L, Schley C, Fletcher K, Nudds S, et al. Intensive youth outreach in mental health: an integrated framework for understanding and intervention. Soc Work Ment Health. 2008;7(1-3):153-75.

62. Bagshaw S. Survey of the network of youth health service providers (NYHSP): affiliated to New Zealand association for adolescent health and development (NZAAHD). N Z Med J. 2006;119(1243):105-11.

63. Monson K, Thurley M. Consumer participation in a youth mental health service. Early Interv Psychiatry. 2011;5(4):381-8.

64. Wade D, Johnston A, Campbell B, Littlefield L. Early intervention services in youth mental health. Clin Psychol. 2007;11(3):108-14.

65. Youth One Stop Shop (YOSS). 2011-2012 annual report. 2012. http:// www.yoss.org.nz. Accessed 21 Sept 2018.

66. Katz M, Urkin J, Bar-David Y, Cohen AH, Warshawsky S, Barak N. Child health care centres: an academic model for comprehensive child health care in the community. Child Care Health Dev. 2005;31(2):217-22.

67. Rowlandson PH, Smith C. An interagency service delivery model for autistic spectrum disorders and attention deficit hyperactivity disorder. Child Care Health Dev. 2009;35(5):681-90.

68. Hopkins G. The Wight stuff. Community Care. 2004;1550:38-9.

69. Callaly T, von Treuer K, van Hamond T, Windle K. Forming and sustaining partnerships to provide integrated services for young people: an overview based on the headspace Geelong experience. Early Interv Psychiatry. 2011;5(Suppl 1):28-33.

70. Rickwood D, Van Dyke N, Telford N. Innovation in youth mental health services in Australia: common characteristics across the first headspace centres. Early Interv Psychiatry. 2015;9(1):29-37.

71. Nadeau L, Jaimes A, Rousseau C, Papazian-Zohrabian G, Germain K, Broadhurst J, et al. Partnership at the forefront of change: documenting the transformation of child and youth mental health services in Quebec. J Can Acad Child Adolesc Psychiatry. 2012;21(2):91-7.

72. Teague GB, Mueser KT, Rapp CA. Advances in fidelity measurement for mental health services research: four measures. Psychiatr Serv. 2012;63(8):765-71.

73. Rickwood D, Mazzer KR, Telford NR, Parker AG, Tanti CJ, McGorry PD. Changes in psychological distress and psychosocial functioning in young people visiting headspace centres for mental health problems. Med J Aust. 2015;202(10):537-42.

74. Muir K, McDermott S, Gendera S, Flaxman S, Patulny R, Sitek T, et al. Independent evaluation of headspace: the National Youth Mental Health Foundation, Interim Evaluation Report, SPRC Report 5/09, report prepared for headspace: National Youth Mental Health Foundation, The University of Melbourne. 2008.

75. CYMHSU Collaborative. Child and youth mental health and substance use (CYMHSU) collaborative update. 2016. 
76. CYMHSU Collaborative. The Charter. 2015.

77. Ontario Centre of Excellence for Child and Youth Mental Health. Evidence in-sight: access to child and youth mental health services. 2015

78. Schley C, Radovini A, Halperin S, Fletcher K. Intensive outreach in youth mental health: description of a service model for young people who are difficult-to-engage and 'high-risk'. Child Youth Serv Rev. 2011;33(9):1506-14

79. Barbic SP, Leon A, Manion I, Irving S, Zivanovic R, Jenkins E, et al. Understanding the mental health and recovery needs of Canadian youth with mental health disorders: a Strategy for Patient-Oriented Research (SPOR) collaboration protocol. Int J Ment Health Syst. 2019;13(1):6.

80. Nicholas A, Holloway E, Telford N, Rickwood D. Development of the headspace Family and Friends Satisfaction Scale: findings from a pilot study. Early Interv Psychiatry. 2018;12(3):478-82.

81. Pomare C, Long JC, Ellis LA, Churruca K, Braithwaite J. Interprofessional collaboration in mental health settings: a social network analysis. J Interprof Care. 2018; https://doi.org/10.1080/13561820.2018.1544550.

82. Morgan S, Pullon S, Garrett S, McKinlay E. Interagency collaborative care for young people with complex needs: front-line staff perspectives. Health Soc Care Community. 2019;27(4):1019-30.

83. Schley C, Ryall V, Crothers L, Radovini S, Fletcher K, Marriage K, et al. Early intervention with difficult to engage, 'high-risk'youth: evaluating an intensive outreach approach in youth mental health. Early Interv Psychiatry. 2008;2(3):195-200.

84. Hilferty F, Cassells R, Muir K, Duncan A, Christensen D, Mitrou F, et al. Is headspace making a difference to young people's lives? Final Report of the independent evaluation of the headspace program. (SPRC report 08/2015). Sydney: Social Policy Research Centre, UNSW Australia; 2015.

85. Gwynne K, Zilibowitz M. Spilstead model of early intervention for vulnerable families. J Paediatr Child Health. 2010;46:6-7.

86. Tobon Jl, Jeffs $L$, editors. Bridging gaps in youth mental health: who is presenting to the youth wellness centre in Hamilton, Ontario? Paper presented at the research and policy in child, youth, and youth adult mental health conference, Tampa, Florida. 2017.

87. LePlatte D, Rosenblum KL, Stanton E, Miller N, Muzik M. Mental health in primary care for adolescent parents. Ment Health Fam Med. 2012;9(1):39-46.

88. Medavie Health Foundation. One-stop access for youth mental health. 2016. http://medaviehealthfoundation.ca/2016/06/one-stop-acces s-for-youth-mental-health/. Accessed 21 Sept 2018.

89. Hawke LD, Mehra K, Settipani C, Relihan J, Darnay K, Chaim G, Henderson J. What makes mental health and substance use services youth friendly? a scoping review of literature. BMC Health Serv. Res. 2019;19(257):2-16. https://doi.org/10.1186/s12913-019-4066-5.

90. McGorry PD, Goldstone SD, Parker AG, Rickwood DJ, Hickie IB. Cultures for mental health care of young people: an Australian blueprint for reform. Lancet Psychiatry. 2014;1 (7):559-68.

91. Hoffmann TC, Glasziou PP, Boutron I, Milne R, Perera R, Moher D, et al. Better reporting of interventions: template for intervention description and replication (TIDieR) checklist and guide. BMJ. 2014;348:g1687.

92. Carbone S, Rickwood D, Tanti C. Workforce shortages and their impact on Australian youth mental health service reform. Adv Ment Health. 2011:10(1):92-7.

93. Purcell $R$, Jorm AF, Hickie IB, Yung AR, Pantelis C, Amminger GP, et al. Demographic and clinical characteristics of young people seeking help at youth mental health services: baseline findings of the Transitions Study. Early Interv Psychiatry. 2015;9(6):487-97.

94. Jorm AF. Australian young people's awareness of headspace, beyondblue and other mental health organizations. Australas Psychiatry. 2009;17(6):472-4

95. Williamson PR, Altman DG, Bagley H, Barnes KL, Blazeby JM, Brookes ST, et al. The COMET handbook: version 1.0. Trials. 2017;18(Suppl 3):280.

96. Bullock H, Waddell K, Lavis JN. Evidence brief: defining the mental health and addictions 'Basket of Core Services' to be publicly funded in Ontario. Hamilton: McMaster Health Forum; 2016.

97. Halsall T, Manion I, Iyer SN, Mathias S, Purcell R, Henderson J. Trends in mental health system transformation: integrating youth services within the Canadian context. Healthc Manag Forum. 2019;32(2):51-5.

98. Tee K, Oyedele O, Salmon A, Helfrich W, Banjo J, Berger M, et al. Foundry - transforming access through integrated youth services in British Columbia, Canada. Early Interv Psychiatry. 2018;12(Suppl. 1):230.
99. Allison S, Bastiampillai T, Goldney R. Australia's national youth mental health initiative: is headspace underachieving? Aust N Z J Psychiatry. 2016:50(2):111-2.

100. Coates D, Howe D. The importance and benefits of youth participation in mental health settings from the perspective of the headspace Gosford Youth Alliance in Australia. Child Youth Serv Rev. 2014;46:294-9.

101. Coates D, Howe D. The design and development of staff wellbeing initiatives: staff stressors, burnout and emotional exhaustion at children and young people's mental health in Australia. Adm Policy Ment Health. 2015;42(6):655-63.

102. Gwinner K. Arts, therapy, and health: three stakeholder viewpoints related to young people's mental health and wellbeing in Australia. Arts Psychother. 2016;50:9-16.

103. Hodges CA, O'Brien MS, McGorry PD. headspace: National Youth Mental Health Foundation: making headway with rural young people and their mental health. Aust J Rural Health. 2007:15(2):77-80.

104. Howe D. ycentral — an integrated youth mental health service platform. Int J Integr Care. 2014;14:1-2.

105. Howe D, Batchelor S, Bochynska K. Finding our way: youth participation in the development and promotion of youth mental health services on the NSW Central Coast. Adv Ment Health. 2011;10(1):20-8.

106. Howe D, Dimopoulos-Bick TL. The CHOICE Project: integrating peer support and shared decision making in a youth mental health service. Int J Integr Care. 2014;14(9):1-2.

107. Muir K, Powell A, McDermott S. 'They don't treat you like a virus': youthfriendly lessons from the Australian National Youth Mental Health Foundation. Health Soc Care Community. 2012;20(2):181-9.

108. Rickwood D, Nicholas A, Mazzer K, Telford N, Parker A, Tanti C, et al. Satisfaction with youth mental health services: further scale development and findings from headspace-Australia's National Youth Mental Health Foundation. Early Interv Psychiatry. 2015;11:296-305.

109. Scott E, Naismith S, Whitwell B, Hamilton B, Chudleigh C, Hickie I. Delivering youth-specific mental health services: the advantages of a collaborative, multi-disciplinary system. Australas Psychiatry. 2009;17(3):189-94.

110. Simmons MB, Parker AG, Hetrick SE, Telford N, Bailey A, Rickwood D. Development of a satisfaction scale for young people attending youth mental health services. Early Interv Psychiatry. 2014;8(4):382-6.

111. Yap MB, Reavley NJ, Jorm AF. Australian youth still have limited awareness of headspace: results from a national survey. Aust N Z J Psychiatry. 2012;46(1):28-34.

112. Cross SP, Hermens DF, Scott J, Salvador-Carulla L, Hickie IB. Differential impact of current diagnosis and clinical stage on attendance at a youth mental health service. Early Interv Psychiatry. 2017;11(3):255-62.

113. Hamilton BA, Naismith SL, Scott EM, Purcell S, Hickie IB. Disability is already pronounced in young people with early stages of affective disorders: data from an early intervention service. J Affect Disord. 2011;131(1-3):84-91.

114. McCann TV, Lubman DI. Young people with depression and their experience accessing an enhanced primary care service for youth with emerging mental health problems: a qualitative study. BMC Psychiatry. 2012;12(1):96

115. McGorry PD, Mei C. Early intervention in youth mental health: progress and future directions. Evid Based Ment Health. 2018;21(4):182-4.

116. Ospina-Pinillos L, Davenport T, Iorfino F, Tickell A, Cross S, Scott EM, et al. Using new and innovative technologies to assess clinical stage in early intervention youth mental health services: evaluation study. J Med Internet Res. 2018;20(9):e259.

117. Schley C, Pace N, Mann R, McKenzie C, McRoberts A, Parker A. The headspace brief interventions clinic: increasing timely access to effective treatments for young people with early signs of mental health problems. Early Interv Psychiatry. 2018; https://doi.org/10.1111/ eip.12729.

118. Scott EM, Hermens DF, Naismith SL, White D, Whitwell B, Guastella AJ, et al. Thoughts of death or suicidal ideation are common in young people aged 12 to 30 years presenting for mental health care. BMC Psychiatry. 2012;12(1):234.

119. Walker S. A description of the establishment of a new child and adolescent mental health service in the United Kingdom. J Child Adolesc Ment Health. 2010;22(1):35-9. 
120. Illback RJ, Bates T. Transforming youth mental health services and supports in Ireland. Early Interv Psychiatry. 2011;5(Suppl 1):22-7.

121. Heenan D, Birrell D. Organisational integration in health and social care: some reflections on the Northern Ireland experience. J Integr Care. 2009;17(5):3-12.

122. James AM. Principles of youth participation in mental health services. Med J Aust. 2007;187(7):S57.

123. Purcell R, Fraser R, Greenwood-Smith C, Baksheev GN, McCarthy J, Reid $D$, et al. Managing risks of violence in a youth mental health service: a service model description. Early Interv Psychiatry. 2012;6(4):469-75.

124. Rice S, Halperin S, Blaikie S, Monson K, Stefaniak R, Phelan M, et al. Integrating family work into the treatment of young people with severe and complex depression: a developmentally focused model. Early Interv Psychiatry. 2018;12(2):258-66.

125. Rousseau C, Laurin-Lamothe A, Nadeau L, Deshaies S, Measham T. Measuring the quality of interprofessional collabroation in child mental health collaborative care. Int J Integr Care. 2011;12:8.
126. Zonneveld R. A one-stop shop for Wellington youth. Nurs N Z. 2014:20(7):28-9.

127. Bailey R, Torrie R, Osborne R, Bagshaw S, Blyth S, Davidson J, et al. How we know what we're doing works. Measuring youth outcomes at Kapiti Youth Support Impact evaluation - Summary report. 2013.

128. Lee VW, Murphy BP. Broadening the early intervention paradigm: a one stop shop for youth. Early Interv Psychiatry. 2013;7(4):437-41.

129. Youth Wellness Hubs Ontario. Youth Wellness Hubs Onatrio: a primer. 2017. https://youthhubs.ca/en/. Accessed 21 Sept 2018.

\section{Publisher's Note}

Springer Nature remains neutral with regard to jurisdictional claims in published maps and institutional affiliations.
Ready to submit your research? Choose BMC and benefit from:

- fast, convenient online submission

- thorough peer review by experienced researchers in your field

- rapid publication on acceptance

- support for research data, including large and complex data types

- gold Open Access which fosters wider collaboration and increased citations

- maximum visibility for your research: over $100 \mathrm{M}$ website views per year

At BMC, research is always in progress.

Learn more biomedcentral.com/submissions 